\title{
Effects of College Students' Perceived Stress on Anxiety in the Time of COVID-19: The Chain Mediation Role of Psychological Resilience and Regulatory Emotional Self-Efficacy
}

\author{
Ting $\mathrm{Li}^{1,3, *}$ and Qiang $\mathrm{Xie}^{2,3}$ \\ ${ }^{1}$ Center for Evaluation and Faculty Development, Hunan First Normal University, Changsha, 410205, China \\ ${ }^{2}$ Teaching Affairs Office, Hunan First Normal University, Changsha, 410205, China \\ ${ }^{3}$ Graduate School of Sehan University, Mokpo, 58613, South Korea \\ *Corresponding Author: Ting Li. Email: pgzxlt@hnfnu.edu.cn \\ Received: 07 September 2021 Accepted: 09 November 2021
}

\begin{abstract}
COVID-19 has changed the way of learning and life of college students. The purpose is to explore the relationship between perceived stress and anxiety, and the mediating effect of resilience and regulatory emotional self-efficacy in college students during COVID-19. 309 students from three colleges were selected as research participants (average age $19.79 \pm 1.11$ years). SPSS was used to measure the correlation between variables. Amos was used to test the path coefficient and mediating effect of the hypothetical model. The results show that: Firstly, perceived stress has a significantly and positively predictive effect on anxiety. Secondly, psychological resilience and regulatory emotional self-efficacy independently played a significant and partially mediating effect between perceived stress and anxiety. Among them, perceived stress had a negative predictive effect on psychological resilience and regulatory emotional self-efficacy. Psychological resilience and regulatory emotional self-efficacy had a negative predictive effect on anxiety. Thirdly, psychological resilience and regulatory emotional self-efficacy play a chain mediation role between psychological stress and anxiety. Regulatory emotional self-efficacy cannot only directly predict the level of anxiety, but also indirectly predict the level of anxiety by regulating psychological resilience. This study reveals the relationship between college students' perceived stress and anxiety, and the mediating effect of psychological resilience and regulatory emotional self-efficacy in the new time of COVID-19. The chain mediation role of psychological resilience and regulatory emotional self-efficacy reminds college mental health educators that improving students' regulatory emotional self-efficacy is an important way to promote students' mental health.
\end{abstract}

\section{KEYWORDS}

Perceived stress; regulatory emotional self-efficacy; psychological resilience; anxiety

\section{Introduction}

The pressure of coronavirus disease (COVID-19) has had a great impact on people's physical and mental health all over the world. Although the epidemic has been partially controlled through global epidemic prevention actions, news reports on the development of the epidemic, travel bans and isolation policies have not only caused public anxiety while controlling the epidemic, but also brought people stress reactions such as psychological pressure, fear and tension to varying degrees [1]. Some scholars have 
proposed that natural disasters, as a stressful event, can have an impact on mental health and may cause symptoms such as anxiety and depression [2]. While COVID-19 has now moved from an explosive growth phase to a normal containment phase, the emotional response to the epidemic is likely to persist long time after the epidemic subsides [3]. The COVID-19 pandemic has brought about changes in the way college students study and socialize, which also poses a threat to their mental health. During the outbreak of COVID-19, college students' negative emotions, such as anxiety and psychological problems are increasingly emerging, and there were different degrees of anxiety and depression [4]. Some scholars surveyed 1667 college students in Shaanxi Province during the epidemic and concluded that $42.2 \%$ of them felt anxious [5]. College students is a large number of special group. They are in the stage of substantial independence with the obvious reduction of direct guidance and supervision from parents [6]. Compared with students in other stages, college students need to face negative emotions such as pressure and anxiety alone [7]. Therefore, research on the stress and emotions of college students during COVID19 can help improve the situation.

Previous studies have shown that chronic stress may lead to anxiety through a variety of mechanisms, and there is a positive correlation between stress and anxiety [8]. Some scholars also suggested that there were many common risk factors and symptoms between stress and anxiety, but the cause of the connection between these psychological syndromes remained to be determined [9]. This is because stress is not only an event that triggers negative emotional response, but a two-way process of interaction between individual and environment. Stress does not necessarily turn into anxiety. Different individuals may face stressful life events differently because of differences in psychological mechanisms. Therefore, studying the psychological transformation mechanism of college students' stress and anxiety can help college students improve their ability to face pressure, avoid anxiety and maintain healthy psychology. In previous studies, the mediating role of psychological resilience or self-efficacy between stress coping and mental health has been proved to varying degrees. As a personality trait, psychological resilience affects individual mental health. People with strong psychological resilience can face pressure and recover from it [10]. At the same time, if student express self-confidence in their action ability, they can also avoid anxiety and promote mental health. Low self-efficacy will lead to negative emotions, such as anxiety, depression, helplessness and decline in academic performance [11].

Especially in time of COVID-19, there is insufficient research on college students' mental health such as stress and anxiety. Based on this, this study aims to explore the influence of psychological resilience and emotional regulation on the relationship between perceived stress and anxiety among college students, and explore the internal connection mechanism of perceived stress and anxiety among college students under the background of COVID-19 prevention and control normalization. Thus, it provides a practical basis for the effective implementation of mental health education for college students in the time of COVID-19. The main problems of this study are as follows:

Firstly, what is the overall level of psychological stress and anxiety of college students in time of COVID-19?

Secondly, does college students' psychological stress have a direct predictive effect on anxiety?

Thirdly, after adding psychological elasticity and emotion regulation self-efficacy to the stress and anxiety influence model, how does the stress of college students affect anxiety?

\section{Literature Review}

\subsection{Relationship between Perceived Stress and Anxiety}

The terms stress and anxiety are common in everyday life, and people have different definitions for

them. These terms are also found in psychological or psychiatric studies by confusion and disagreement. Some scholars believe that stress is a cognitive process in which people react to external things, and 
stress is closely related to people's coping ability. While anxiety is an unpleasant emotional state, anxiety is more related to people's instinctive reaction [12]. Pressure is divided into single pressure, superimposed pressure and destructive pressure according to strength. Based on the characteristics of the time of COVID-19, college students' stress is a chronic stress in life or study caused by the epidemic, which belongs to the category of unitary stress. Because different college students have different perceptions of stress, it is possible to show different emotional states to the same stress. Therefore, this study explores the effect of college students' perceived stress on anxiety. Perceived stress is the evaluation process of stress. Individuals can evaluate whether a life event causes stress to themselves only by giving stimulus events a certain meaning through the evaluation process of perception. Perceived stress is a subjective response to specific stress [13]. Psychological research has shown that various stimulus events and unfavorable factors in life can cause psychological confusion or threats to people. When individuals perceive pressure, they will show different psychological reactions and become perceived stress. Prior research has shown that there is a direct predictive relationship between stress, perceived stress, and anxiety. Scholars explored the impact of stress on anxiety in different populations during the COVID-19 epidemic and found that stress is directly or indirectly related to anxiety [8]. For example, the stress perception of primary and secondary school teachers directly affects the anxiety level, and indirectly affects the anxiety level through coping style [14]. Anxiety disorder and depression are positively correlated with all dimensions of stress and negative coping [15]. There is a significant interaction between the perceived stress of isolated persons, anxiety sensitivity, and the severity of overall anxiety symptoms [16].

Therefore, this study puts forward the hypothesis:

H1. College students'stress perception has a positive impact on college students' anxiety level.

\subsection{The Mediating Role of Psychological Resilience}

Resilience has a multidimensional feature, which can change with the environment, age, experience and cultural background. Mental toughness plays an important role for college students, which can help them better cope with the changes brought by the epidemic and recover their mental health through selfadjustment. In terms of results, resilience refers to the development of individual adaptability after experiencing serious threats [17]. In terms of process, resilience is an adaptive process, which refers to the dynamic process of active adaptation and development in adversity [18], which can help individuals deal with stress stimuli and finally reach the adaptive level. In terms of quality, resilience is regarded as a quality and ability of individuals to withstand changes and adapt to changing environments. Brooks defined psychological resilience as the ability of individuals to recover quickly after injury and return to the pre-crisis state [19]. From these definitions, it is not difficult to see that resilience has become an important psychological mechanism and personal quality when dealing with environmental pressure. With the interest of positive psychology, the effect of resilience on stress or mental health has been confirmed. Studies have found that psychological resilience is very important in coping with common environmental stress, conflict, uncertainty and extreme adversity [20]. There was a significant negative correlation between perceived stress and mental resilience. Individuals with high mental resilience can adjust themselves and relieve pressure when facing pressure, while individuals with low mental resilience are more prone to anxiety and depression symptoms due to their poor ability to resist adversity. The strength of psychological resilience directly affects the level of anxiety and depression [21].

In addition, some studies have proved that there was a significant negative correlation between the dimensions of psychological resilience and anxiety. Sang et al. [22] divided the students into two groups and found that improving psychological resilience reduced the level of state anxiety. After Psychological resilience group counseling intervention, the level of students' psychological resilience has been significantly improved, and the effects of intervention on resilience, state anxiety and bullying can last until 6 months after the end of the intervention. In the COVID-19 investigation, Zhou [23] investigated 
the anxiety level of college students and found that the anxiety level of students was significantly higher than that of ordinary people, and the psychological resilience of college students could negatively predict anxiety.

In addition, based on the study of the relationship between stress and resilience and the relationship between resilience and anxiety, researchers have also begun to study the mediating role of resilience. Some scholars have proved that psychological resilience played a partial mediating role between depression and all burnout dimensions [24]. Psychological resilience mediates the relationship between fear of COVID19 and stress [25]. Other studies have confirmed that stress may be a risk factor for anxiety and depression symptoms, and psychological resilience has a protective effect on anxiety and depression symptoms, and psychological resilience plays a partial mediating role [26]. The mediating effect of mental resilience between mental stress and mental health has been confirmed by more and more studies.

Based on this, this study puts forward the following three hypotheses:

H2. College students' psychological stress negatively affects their psychological resilience;

H3. Psychological resilience negatively affects the level of anxiety;

H4. Psychological resilience plays an intermediary role between college Students' psychological stress and anxiety.

\subsection{The Mediating Effect of Regulatory Emotional Self-Efficacy}

The famous psychologist Bandura first put forward the concept of self-efficacy, which refers to the confidence in one's ability to act in this way or produce desired results. He defined regulatory emotional self-efficacy as a kind of self-confidence and management ability in the emotional field. It is an individual's evaluation of whether he can effectively regulate his emotional state. It will directly or indirectly affect various social and psychological functions [27]. Overall, emotion regulation self-efficacy is the ability of self-efficacy in emotion control. At the same time, the researchers also discussed the relationship between stress and self-efficacy. Some scholars maintained that psychological stress was negatively correlated with self-efficacy [28], some scholars advocated that self-efficacy should be studied according to the different nature of stress. Challenging stress is conducive to enhance self-efficacy, which is significantly positively correlated with self-efficacy, while obstructive stress has the opposite effect [29], some scholars also believe that both challenging stress and obstructive stress negatively predict self-efficacy [30].

Although there are disagreements on the correlation between stress and self-efficacy, the relationship between regulatory emotional self-efficacy on negative emotions such as anxiety and depression is basically the same. It is considered that regulatory emotional self-efficacy has a negative predictive effect on negative emotions such as depression and anxiety [31]. College students with higher regulatory emotional self-efficacy showed lower depression level, less negatively emotions and higher psychological well-being [32].

In recent years, more and more studies have focused on the mediating role of self-efficacy between stress and mental outcomes. For example, some studies demonstrated the mediating role of self-efficacy between stress and anxiety, and Self-efficacy mediates the effects of stressful life events or daily stressors on positive and negative mental health [33]. McLaughlin and Hatzenbuehler studied the relationship between adverse emotion regulation and stress and depressive symptoms. They found that although stress had no direct effect on subsequent depressive symptoms, maladaptive emotional regulation mediated the relationship between stress and depressive symptoms, and emotional regulation played an intermediary role [34].

Based on this, this study puts forward the following three hypotheses:

H5. College students' psychological pressure negatively affects their regulatory emotional self-efficacy;

H6. Regulatory emotional self-efficacy negatively affects the level of anxiety;

H7. Regulatory emotional self-efficacy plays an intermediary role between college students' psychological stress and anxiety. 


\subsection{The Chain Mediating Effect of Psychological Resilience and Regulatory Emotional Self-Efficacy}

For the relationship between the two mediating variables in this study, some studies have confirmed that there is a significant positive correlation between regulatory emotional self-efficacy and psychological resilience [35]. Individuals with higher levels of regulatory emotional self-efficacy can better express positive emotions and enhance their mental resilience in the face of stress. In addition, studies have shown that self-efficacy has no significant effect on depression, and self-efficacy has a significant effect on depression through psychological resilience [36]. It can be seen that self-efficacy and psychological resilience have a chain mediating effect on bad emotions. Liu [37] investigated the relationship between emotional intelligence and coping styles of middle school students and found that self-efficacy can not only predict coping styles through psychological resilience, but also directly affect coping styles. Psychological resilience and self-efficacy have a double mediating effect in it. These studies have proved that self-efficacy and psychological resilience can simultaneously produce mediating effects. But no research has shown how they work together between stress and anxiety. But there is no research to prove how they work simultaneously between stress and anxiety. Therefore, this paper puts forward the following assumptions:

H8. Psychological resilience and regulatory emotional self-efficacy play a chain intermediary role between psychological stress and anxiety.

The hypothetical conceptual model is shown in the Fig. 1.

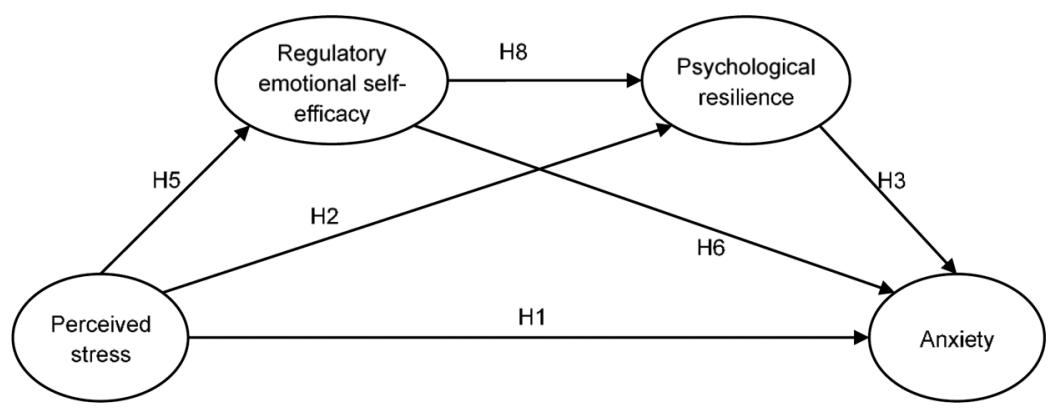

Figure 1: Research hypothesis model

\section{Research Methods}

\subsection{Research Participants}

Random sampling was used in this study. During the period from June 05 to June 25, 2021, an electronic questionnaire was distributed to three undergraduate colleges in Hunan. The research participants are from Grades 1-4 students in three colleges. Before issuing the questionnaire, the researcher introduced the research purpose and data collection methods to the participants, and informed the participants that the completed questionnaire would be kept confidential, and they also had the right to refuse to participate in the study. In order to ensure that the participants can fill in carefully and accurately, the researchers conducted a brief interview with all participants, and fed back the scores of the psychological test questionnaire to the participants after filling in.

\subsection{Research Participants}

\subsubsection{Perceived Stress Scale (PSS)}

The Chinese version of Perceived Stress Scale (PSS) translated and revised by Yang et al. [38,39] was used in this study. The PSS includes two factors: sense of loss of control and tension. The PSS contains 14 items, including 7 positive items and 7 negative items, all of which carry a 4-point range of responses, 
as follows: " 0 =never, $1=$ almost never, $2=$ sometimes, $3=$ fair often, $4=$ very often". Coefficient alpha reliability for the PSS was 0.85 , the test-retest correlation was 0.85 .

\subsubsection{The Connor-Davidson Resilience Scale (CD-RISC)}

The Chinese version of The Connor-Davidson Resilience Scale (CD-RISC) jointly developed by Connor and Davidson was used in this study. The Chinese version of CD-RISC was translated and revised by Connor et al. [40,41], including three factors: tenacity, self-improvement and optimism. The CD-RISC contains 25 items, all of which carry a 5-point range of responses, as follows: " $1=$ not true at all, 2 = rarely true, $3=$ sometimes true, $4=$ often true, $5=$ true nearly all of the time". The internal consistency coefficient of the Chinese version of the scale is 0.91 . The three-factor structure (tenacity, self-improvement and optimism) is reasonable. CD-RISC can reflect the recovery ability of different populations and has good psychometric characteristics.

\subsubsection{Generalized Anxiety Disorder Scale (GAD-7)}

This study used the Generalized Anxiety Disorder Scale (GAD-7) compiled by Spitzer et al. [42]. GAD7 is a self-report scale for measuring anxiety symptoms, which is simple and practical. It contains 7 items, all of which carry a 4-point range of responses, as follows: " $0=$ none at all, $1=$ sometimes, $2=$ most of the time, $3=$ almost every day". With the increase of scores, anxiety symptoms worsen. In Spitzer version, GAD-7 was greater than or equal to 10 points as the boundary value for screening GAD. The scores of 5, 10, and 15 respectively represent the level of mild, moderate, and severe anxiety. The internal consistency of the GAD-7 was excellent (Cronbach's $\alpha=0.92$ ). Test-retest reliability was also good $(\mathrm{ICC}=0.83$ ).

\subsubsection{Regulatory Emotional Self-Efficacy Scale (RES)}

The Chinese version of Regulatory Emotional Self-efficacy scale (RES), which is compiled by Caprara was used in this study [43]. The Chinese version was translated and revised by Wen et al. [44]. The Chinese version of RES includes 3 factors: expressing positive emotional self-efficacy (POS), regulating depression/ pain emotional self-efficacy (DES) and regulating anger/anger emotional self-efficacy (ANG). The RES contains 25 items, all of which carry a 5-point range of responses. The internal consistency reliability of RES is well (Cronbach's $\alpha=0.91$ ), and the internal consistency reliability of the three dimensions is also well (Cronbach's $\alpha>0.8$ ). The Chinese version of RES has high reliability.

\subsection{Data Analysis}

Firstly, the frequency and percentage were used to analyze the sample characteristics of participants. Secondly, the Common Method Bias (CMB) is tested by controlling for the effects of unmeasured latent method factor. At the same time, in order to ensure the reliability and validity of the applied scales, SPSS 23.0 was used to test the reliability and validity of the four scales. Furthermore, the sample characteristics were analyzed by mean and standard deviation, and the correlation matrix and descriptive statistics were measured by SPSS 23.0. Finally, Amos 23.0 is used to carry out confirmatory factor analysis, path coefficient test and intermediary effect test on the hypothetical model. Among them, the intermediary effect test uses the nonparametric percentile bootstrap estimation method with deviation correction to randomly repeat sampling 2000 times for the sample $(n=309)$.

\section{Results}

\subsection{Demographic Survey Results}

This study was conducted at three college in China from June 15 to 25, 2021. The participants were undergraduates in their first to fourth years of college. A total of 360 questionnaires were sent out and 330 were collected. After eliminating the obvious unreasonable questionnaires, 309 valid questionnaires were obtained and the effective rate was $93.64 \%$. In this survey, female students account for 171 and male students account for 138. The grade distribution of participants was as follows: freshman accounted 
for 72 , sophomore accounted for 86 , junior accounted for 76 , and graduates accounted for 75 . The average age of the students was $19.79 \pm 1.11$ years. The specific demographic characteristics are shown in Table 1 .

Table 1: Demographic characteristics

\begin{tabular}{llll}
\hline \multicolumn{2}{c}{ Category } & Number (n) & Percentage (\%) \\
\hline \multirow{2}{*}{ Gender } & Male & 138 & 44.7 \\
& Female & 171 & 55.3 \\
\hline \multirow{2}{*}{ Grade } & Grade 1 & 72 & 23.3 \\
& Grade 2 & 86 & 27.8 \\
& Grade 3 & 76 & 24.6 \\
& Grade 4 & 75 & 24.3 \\
\hline
\end{tabular}

\subsection{Common Method Bias}

Following Podsakoff's suggestion, the Common Method Bias (CMB) is tested by controlling for the effects of unmeasured latent method factor [45]. Based on the original nine trait factors, taking all items as the indicators of method factors, a two-factor model is established. The fitting index of the two-factor model is: $x^{2} / d f=22.683$, TLI $=0.843, \mathrm{CFI}=0.854$, RMSEA $=0.132$. The model fit is poor, the difference between the two-factor model and the trait model is not significant, and it is generally considered that the deviation of the common method is not serious.

\subsection{Reliability and Validity Test}

In order to ensure the reliability and validity of the applied scales, the reliability and validity of the four scales were tested respectively. Cronbach's $\alpha$ values of PSS is 0.959 , Cronbach's $\alpha$ values of each factor are: feeling out of control (0.950) and tension (0.933); Cronbach's $\alpha$ values of CD-RISC is 0.979, Cronbach's $\alpha$ values of each factor are: Tenacity (0.967), self-improvement (0.949) and optimism (0.949); Cronbach's $\alpha$ values of RES is 0.955 , Cronbach's $\alpha$ values of each factor are: POS (0.955), DES (0.946) and ANG (0.931). It can be seen that the four scales have good reliability (see Table 2). Secondly, the validity of the scale was tested by confirmatory factor analysis. The TLI and CFI values in the four scales are greater than 0.90 and the RMSEA values are less than 0.08 , it can be considered that the hypothetical model has a very good fit with the research data, so the scale has good validity.

Table 2: Reliability and validity test results

\begin{tabular}{lllllll}
\hline Scale & Factor & Item & $\lambda$ & C.R $(\mathrm{t})$ & AVE & Cronbach's $\alpha$ \\
\hline PSS & Feeling out of control & 7 & $0.816-0.880$ & 0.956 & 0.758 & 0.950 \\
& Feeling tension & 7 & $0.800-0.838$ & 0.955 & 0.751 & 0.933 \\
\hline \multirow{2}{*}{ CD-RISC } & Tenacity & 13 & $0.783-0.876$ & 0.962 & 0.603 & 0.967 \\
& Self-improvement & 8 & $0.762-0.876$ & 0.936 & 0.646 & 0.949 \\
& Optimism & 4 & $0.891-0.921$ & 0.933 & 0.778 & 0.949 \\
\hline \multirow{2}{*}{ GAD-7 } & Anxiety & 7 & $0.740-0.826$ & 0.953 & 0.742 & 0.925 \\
\hline \multirow{2}{*}{ RES } & POS & 4 & $0.896-0.939$ & 0.942 & 0.804 & 0.955 \\
& DES & 4 & $0.885-0.921$ & 0.947 & 0.816 & 0.946 \\
& ANG & 4 & $0.826-0.918$ & 0.926 & 0.759 & 0.931 \\
\hline
\end{tabular}




\subsection{Descriptive Statistics and Correlation Analysis}

Through the independence test and common method deviation test, the research model is a more effective model, so this paper carries on the follow-up research on this basis. Table 3 summarizes the correlation matrix and descriptive statistics between the variables.

Table 3: Correlation matrix and reliability of each dimension $(n=309)$

\begin{tabular}{lllllll}
\hline Factor & $\mathrm{M}$ & $\mathrm{SD}$ & 1 & 2 & 3 & 4 \\
\hline 1. Perceived stress & 1.897 & 0.710 & - & & & \\
2. Regulatory emotional self-efficacy & 3.189 & 0.872 & $-0.244^{* *}$ & - & & \\
3. Psychological resilience & 3.207 & 0.937 & $-0.464^{* *}$ & $0.541^{* *}$ & - & \\
4. Anxiety & 0.940 & 0.635 & $0.619^{* *}$ & $-0.533^{* *}$ & $-0.684^{* *}$ & - \\
\hline
\end{tabular}

Notes: ${ }^{*} p<0.05 ; * * p<0.01$.

Among the factors of perceived stress, regulatory emotional self-efficacy, psychological resilience and anxiety, the standard deviation of anxiety is lowest. The standard deviation of anxiety is low, indicating that the subjects' perception difference of anxiety is the smallest. This may be related to the fact that the subjects live in a similar campus environment. The mean and standard deviation of regulatory emotional self-efficacy and psychological resilience are not much different, indicating that they are at a similar level. The deviation value $(-0.863 \sim 0.981)$ and kurtosis value $(-0.780 \sim 0.904)$ of the four factors are in the ideal range, and the correlation coefficient and significance between the factors meet the statistical requirements. The data collected in this survey are suitable for data analysis.

\subsection{Test of Structural Equation Model}

Amos 23.0 was used to test the fitting degree between the measurement model and the actual data. The results show that, $x^{2} / d f=1.578, \mathrm{TLI}=0.982, \mathrm{CFI}=0.985, \mathrm{RMSEA}=0.043(0.029 \sim 0.057)$. It shows that the measurement model meets the ideal standard, and the structural model can be further tested. If the correlation between independent variables is very high, there may be a multicollinearity problem.

Calculated according to the formula $V I F_{j}=1 /\left(1-R_{j}^{2}\right)\left(R_{j}^{2}\right.$ is the coefficient of determination, the measurement coefficient obtained by regression with other independent variables; variance inflation factor, VIF), The VIF of all predictive variables in this study is not higher than 5, so it can be considered that there is no multicollinearity problem.

In order to explore the relationship between perceived stress and anxiety, and the mediating role of psychological resilience and regulatory emotional self-efficacy in this process, this study first tested the direct predictive effect of stress on anxiety. The results show that the model fits the data well: $x^{2} / d f=1.978, \mathrm{TLI}=0.980, \mathrm{CFI}=0.986, \mathrm{RMSEA}=0.056$. Stress can significantly predict anxiety $(\beta=0.692$, $P<0.001$ ), hypothesis 1 is supported.

This research then builds a chain mediation model of regulatory emotional self-efficacy and psychological resilience. The results show that the mediating model of regulatory emotional self-efficacy and resilience has a good fit index (see Table 4). Perceived stress negatively affects regulatory emotional self-efficacy $(\beta=-0.28, p<0.001)$ and mental toughness $(\beta=-0.37, p<0.001)$. Hypothesis 2 and Hypothesis 5 are supported. Second, regulatory emotional self-efficacy negatively significantly affects anxiety $(\beta=-0.27, p<0.001)$, resilience also negatively affects anxiety $(\beta=-0.35, p<0.001)$, Hypothesis 3 and Hypothesis 6 Get support. The specific path relationship between the variables in the research model is shown in Fig. 2. 
Table 4: The model's fit indices

\begin{tabular}{lllllll}
\hline$\chi^{2}$ & df & $x^{2} / d f$ & CFI & NFI & TLI & RMSEA \\
\hline $132.581 * * *$ & 84 & 1.587 & 0.985 & 0.961 & 0.982 & 0.043 \\
\hline
\end{tabular}

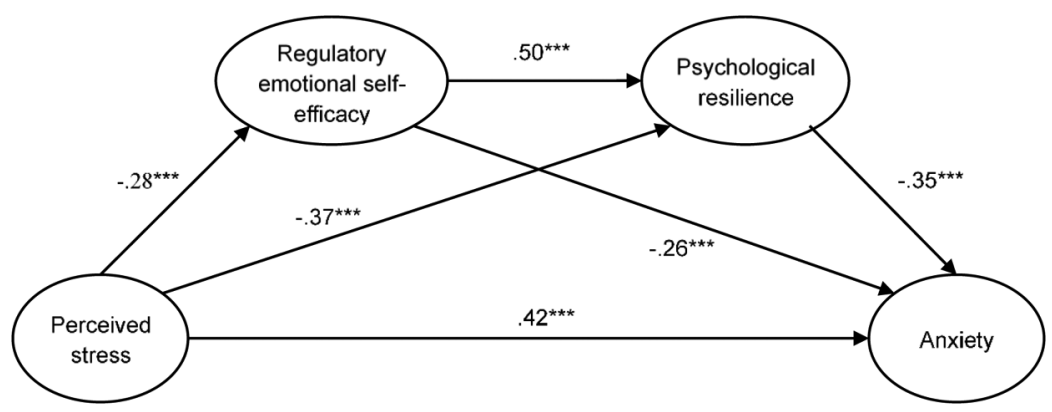

Figure 2: SEM of regulatory emotional self-efficacy and psychological resilience

\subsection{Test of Mediating Effect}

In order to ensure the consistency and stability of the analysis results, this study adopted the biascorrected bootstrap estimation method. The sample $(n=309)$ was randomly selected for 2000 times, shown in Table 5.

Table 5: Results of bootstrap test of mediating effect

\begin{tabular}{lllc}
\hline Impact path & $\begin{array}{l}\text { The mediating effect } \\
\text { of standardization }\end{array}$ & \multicolumn{2}{c}{$\begin{array}{c}95 \% \text { Confidence } \\
\text { interval }\end{array}$} \\
\cline { 2 - 4 } & & $\begin{array}{l}\text { Lower } \quad \begin{array}{l}\text { Upper } \\
\text { limit }\end{array} \\
\text { limit }\end{array}$ \\
\hline $\begin{array}{l}\text { Perceived stress } \rightarrow \text { Regulatory emotional self-efficacy } \\
\rightarrow \text { Anxiety }\end{array}$ & $-0.28^{*-0}-0.26=0.07$ & 0.02 & 0.16 \\
Perceived stress $\rightarrow$ Psychological resilience $\rightarrow$ Anxiety & $-0.37 *-0.35=0.13$ & 0.06 & 0.23 \\
Perceived stress $\rightarrow$ Regulatory emotional self-efficacy & $-0.28^{*} 0.50^{*-0}-0.35=$ & 0.02 & 0.08 \\
$\rightarrow$ Psychological resilience $\rightarrow$ Anxiety & 0.05 & & \\
\hline
\end{tabular}

Table 5 shows the standardized estimates of each indirect path and the $95 \%$ confidence interval of the mediation effect. If the $95 \%$ confidence interval does not contain 0 , the mediation effect is significant. According to Table 5, the $95 \%$ confidence intervals of the three indirect paths do not contain 0 , indicating that the mediating effect of self-efficacy is significant; The mediating effect of psychological resilience was significant; The chain mediation effect was significant. Therefore, Hypothesis 4, Hypothesis 7 and Hypothesis 8 of this study are valid.

\section{Conclusions and Discussion}

The purpose of this study was to explore the mediating role of regulatory emotional self-efficacy and resilience in college students' perceived stress and anxiety, and to understand the internal mechanism of college students' perceived stress and anxiety in the time of COVID-19. Based on the results of the research, this study provided several conclusions. 
First, the results of this study confirm Hypothesis 1 and are basically consistent with previous studies. It is found that there is a positive correlation between college students' stress perception and anxiety level. This tells the teachers of colleges and universities that they should help college students to release and mediate the pressure in life and study through various methods, or to cultivate the ability of college students to resist pressure through mental health education [46]. In order to prevent stress from turning into depression and anxiety, which affects the normal development of college students' personality and behavior. According to the interview with the participants during the questionnaire, the stress of college students is mainly reflected in the sudden increase in academic pressure after returning to school and the employment pressure caused by the epidemic. Some students feel academic pressure because they fail to carry out online learning well during online learning and fail to adjust their learning state in time after returning to school, resulting in disconnection from the progress of school education. Junior and senior students fear that the recurrence of the epidemic will make the employment competition more intense, and feel pressure on the possible pressure of graduation, namely unemployment. These common stress problems of college students caused by the epidemic are also consistent with Ren's research conclusion [47]. Therefore, although the mean of the stress of college students still belongs to the normal range, timely intervention and guidance are still essential.

Second, this study confirms the research Hypotheses 2, 3 and 4, and is consistent with the previous research results. It is found that psychological resilience plays a partial and significant intermediary role in perceived and anxiety, which shows that psychological resilience is the key factor in understanding stress and predicting anxiety. When college students face heavy pressure, they will consume their own energy to effectively deal with needs. This process is accompanied by the decline of coping ability, such as psychological resilience [48]. Therefore, the perceived pressure of college students negatively affects psychological resilience. Improving psychological resilience can significantly reduce the anxiety level [22], and college students' psychological resilience has a negative impact on the anxiety level. Based on this, psychological resilience can be said to be an important goal of psychological intervention in public health emergencies [49]. Therefore, we should implement effective psychological support and psychological counseling for college students, improve their psychological resilience, reduce their uncertain impact on the epidemic, and make them feel psychological support, so as to help them deal with the threat of various bad emotions.

Third, this study confirms Hypotheses 5, 6 and 7. It is found that college students' perceived stress will negatively predict self-efficacy, which is consistent with the conclusions of some previous studies [30]. This may be because the thought of college students is not mature enough and their will is relatively weak. Perceived stress will erode and hurt their regulatory emotional self-efficacy. This phenomenon can be explained by resource conservation theory [50]. Emotional loss will threaten people's existing resources, so we need to pay more energy to prevent further loss, but it also further accelerates the loss of resources. Therefore, stress is easy to make people pay energy to deal with. Continuous or long-term stress will accelerate psychological loss, easily produce negative self-awareness, and have a negative impact on regulatory emotional self-efficacy [51]. However, this does not prevent high regulatory emotional selfefficacy from effectively alleviating individual anxiety, fear and other negative emotions in stressful situations.

Fourth, this research further proves Hypothesis 8. Studies have found that perceived stress can predict anxiety levels through the chain mediation of regulatory emotional self-efficacy and resilience. This means that when college students are under pressure, if they want to maintain a certain level of psychological resilience to reduce the incidence of anxiety, they can use the personality trait of regulatory emotional self-efficacy to affect the level of psychological resilience. Regulatory emotional self-efficacy can not only directly predict anxiety level, but also indirectly predict anxiety level by adjusting mental flexibility. Individuals with high regulatory emotional self-efficacy can usually use appropriate methods to solve 
problems, thereby increasing the individual's successful experience, and can also self-control and regulate their own negative emotions. At the same time, it can regulate its own negative emotions to maintain a positive and optimistic mental state, thereby promoting the psychological resilience. To some extent, the chain intermediary role reminds college mental health educators that improving students' regulatory emotional self-efficacy can be an important way to promote students to maintain good mental health.

\section{Implications and Limitations}

The study will help colleges to recognize the possible negative effects of psychological perceived pressure on college students' mental health. In the time of COVID-19, colleges should strengthen students' psychological combing and mental health education, and establish scientific methods to prevent bad emotions. The research focusing on the secondary consequences of the epidemic has made progress, which will also provide research ideas for other scholars to carry out similar research.

The results of this study should be evaluated in the context of its limitations. This study is limited to the impact of stress on anxiety of college students under the background of epidemic normalization. Because of the epidemic situation, this study only collected data in three undergraduate colleges. The object has certain limitations. The impact of these factors on the research results should be considered. At the same time, this study does not distinguish between urban students and rural students. Because there may be differences between rural students and urban students in family support, technical support and social support, it is necessary to make a distinction in the follow-up study. In addition, in terms of data analysis, a variety of methods should be used to study, focusing on whether there are differences in psychological stress and anxiety among different categories of college students, which may provide an important reference for determining the target population of mental health education.

Funding Statement: The authors received no specific funding for this study.

Conflicts of Interest: The authors declare that they have no conflicts of interest to report regarding the present study.

\section{References}

1. Bao, Y., Sun, Y., Meng, S., Shi, J., Lu, L. (2020). 2019-nCoV epidemic: Address mental health care to empower society. The Lancet, 395(10224), e37-e38. DOI 10.1016/S0140-6736(20)30309-3.

2. Kopala-Sibley, D. C., Kotov, R., Bromet, E. J., Carlson, G. A., Danzig, A. P. et al. (2016). Personality diatheses and hurricane sandy: Effects on post-disaster depression. Psychological Medicine, 46(4), 865-875. DOI 10.1017/ S0033291715002378.

3. Hoyt, L. T., Cohen, A. K., Dull, B., Castro, E. M., Yazdani, N. (2021). "Constant stress has become the new normal": Stress and anxiety inequalities among US college students in the time of COVID-19. Journal of Adolescent Health, 68(2), 270-276. DOI 10.1016/j.jadohealth.2020.10.030.

4. Cao, W., Fang, Z., Hou, G., Han, M., Xu, X. et al. (2020). The psychological impact of the COVID-19 epidemic on college students in China. Psychiatry Research, 287, 112934. DOI 10.1016/j.psychres.2020.112934.

5. Yang, Y., Wang, Y., Li, S., Lei, X., Yang, F. (2020). Psychological reaction and influencing factors of Shaanxi college students during the epidemic period of new coronavirus pneumonia. Chinese Journal of School Health, 41(5), 664-667. DOI 10.16835/j.cnki.1000-9817.2020.05.008.

6. Bernier, A., Larose, S., Whipple, N. (2005). Leaving home for college: A potentially stressful event for adolescents with preoccupied attachment patterns. Attachment \& Human Development, 7(2), 171-185. DOI 10.1080/ 14616730500147565.

7. Towbes, L. C., Cohen, L. H. (1996). Chronic stress in the lives of college students: Scale development and prospective prediction of distress. Journal of Youth and Adolescence, 25(2), 199-217. DOI 10.1007/BF01537344.

8. Ross, R. A., Foster, S. L., Ionescu, D. F. (2017). The role of chronic stress in anxious depression. Chronic Stress, 1. DOI 10.1177/2470547016689472. 
9. Ramón-Arbués, E., Gea-Caballero, V., Granada-López, J. M., Juárez-Vela, R., Pellicer-García, B. et al. (2020). The prevalence of depression, anxiety and stress and their associated factors in college students. International Journal of Environmental Research and Public Health, 17(19), 7001. DOI 10.3390/ijerph17197001.

10. Doyle, N., MacLachlan, M., Fraser, A., Stilz, R., Lismont, K. et al. (2016). Resilience and well-being amongst seafarers: Cross-sectional study of crew across 51 ships. International Archives of Occupational and Environmental Health, 89(2), 199-209. DOI 10.1007/s00420-015-1063-9.

11. Gibbons, C. (2010). Stress, coping and burn-out in nursing students. International Journal of Nursing Studies, 47(10), 1299-1309. DOI 10.1016/j.ijnurstu.2010.02.015.

12. Jungbluth, C., MacFarlane, I. M., Veach, P. M., LeRoy, B. S. (2011). Why is everyone so anxious? An exploration of stress and anxiety in genetic counseling graduate students. Journal of Genetic Counseling, 20(3), 270-286. DOI 10.1007/s10897-010-9348-3.

13. Rabaglietti, E., Lattke, L. S., Tesauri, B., Settanni, M., de Lorenzo, A. (2021). A balancing act during COVID-19: Teachers' self-efficacy, perception of stress in the distance learning experience. Frontiers in Psychology, 12, 1-8. DOI 10.3389/fpsyg.2021.644108.

14. Kahn, R. L., Wolfe, D. M., Quinn, R. P., Snoek, J. D., Rosenthal, R. A. (1964). Organizational stress: Studies in role conflict and ambiguity. John Wiley.

15. Nanjundaswamy, M. H., Pathak, H., Chaturvedi, S. K. (2020). Perceived stress and anxiety during COVID19 among psychiatry trainees. Asian Journal of Psychiatry, 54, 102282. DOI 10.1016/j.ajp.2020.102282.

16. Xu, C., Xu, Y., Xu, S., Zhang, Q., Liu, X. et al. (2020). Cognitive reappraisal and the association between perceived stress and anxiety symptoms in COVID-19 isolated people. Frontiers in Psychiatry, 11, 1-8. DOI $10.3389 /$ fpsyt.2020.00858.

17. Gucciardi, D. F., Hanton, S., Gordon, S., Mallett, C. J., Temby, P. (2015). The concept of mental toughness: Tests of dimensionality, nomological network, and traitness. Journal of Personality, 83(1), 26-44. DOI 10.1111/ jopy.12079.

18. Luthar, S. S., Cicchetti, D., Becker, B. (2000). The construct of resilience: A critical evaluation and guidelines for future work. Child Development, 71(3), 543-562. DOI 10.1111/1467-8624.00164.

19. Brooks, S., Amlot, R., Rubin, G. J., Greenberg, N. (2020). Psychological resilience and post-traumatic growth in disaster-exposed organisations: Overview of the literature. BMJ Military Health, 166(1), 52-56. DOI 10.1136/ jramc-2017-000876.

20. Lupe, S. E., Keefer, L., Szigethy, E. (2020). Gaining resilience and reducing stress in the age of COVID-19. Current Opinion in Gastroenterology, 36(4), 295-303. DOI 10.1097/MOG.0000000000000646.

21. García-León, M. Á., Pérez-Mármol, J. M., Gonzalez-Pérez, R., del Carmen García-Ríos, M., Peralta-Ramírez, M. I. (2019). Relationship between resilience and stress: Perceived stress, stressful life events, HPA axis response during a stressful task and hair cortisol. Physiology \& Behavior, 202, 87-93. DOI 10.1016/j. physbeh.2019.02.001.

22. Sang, Q., Li, H. L., Liu, S., Shu, S., Liu, Z. (2019). The effect of resilience group counseling on state anxiety of bullied pupils. Studies of Psychology and Behavior, 17(3), 333.

23. Zhou, Y. (2021). Relationship between resilience and anxiety of college students during the COVID-19 epidemic. Journal of Heilongjiang Institute of Teacher Development, 37(3), 115-118.

24. Serrão, C., Duarte, I., Castro, L., Teixeira, A. (2021). Burnout and depression in Portuguese healthcare workers during the COVID-19 pandemic - the mediating role of psychological resilience. International Journal of Environmental Research and Public Health, 18(2), 636. DOI 10.3390/ijerph18020636.

25. Peker, A., Cengiz, S. (2021). COVID-19 fear, happiness and stress in adults: The mediating role of psychological resilience and coping with stress. International Journal of Psychiatry in Clinical Practice, 25, 1-9. DOI 10.1080/ 13651501.2021.1937656.

26. Shen, X., Yang, Y. L., Wang, Y., Liu, L., Wang, S. et al. (2014). The association between occupational stress and depressive symptoms and the mediating role of psychological capital among Chinese university teachers: A crosssectional study. BMC Psychiatry, 14(1), 1-8. DOI 10.1186/s12888-014-0329-1. 
27. Bandura, A., Caprara, G. V., Barbaranelli, C., Gerbino, M., Pastorelli, C. (2003). Role of affective self-regulatory efficacy in diverse spheres of psychosocial functioning. Child Development, 74(3), 769-782. DOI 10.1111/14678624.00567.

28. Xiong, H., Yi, S., Lin, Y. (2020). The psychological status and self-efficacy of nurses during COVID-19 outbreak: A cross-sectional survey. INQUIRY: The Journal of Health Care Organization, Provision, and Financing, 57. DOI $10.1177 / 0046958020957114$

29. Travis, J., Kaszycki, A., Geden, M., Bunde, J. (2020). Some stress is good stress: The challenge-hindrance framework, academic self-efficacy, and academic outcomes. Journal of Educational Psychology, 112(8), 1632. DOI 10.1037/edu0000478.

30. Li, J., Zhang, X., Wang, X. (2020). How challenge-hindrance stresses influence kindergarten teachers' selfefficacy: A moderated mediation model. Studies in Early Childhood Education, 34(12), 55-67. DOI 10.13861/ j.cnki.sece.2020.12.006.

31. Pu, J., Hou, H., Ma, R. (2017). Direct and indirect effects of self-efficacy on depression: The mediating role of dispositional optimism. Current Psychology, 36(3), 410-416. DOI 10.1007/s12144-016-9429-z.

32. Goldin, P. R., Ziv, M., Jazaieri, H., Werner, K., Kraemer, H. et al. (2012). Cognitive reappraisal self-efficacy mediates the effects of individual cognitive-behavioral therapy for social anxiety disorder. Journal of Consulting and Clinical Psychology, 80(6), 1034. DOI 10.1037/a0028555.

33. Choi, Y. J. (2012). Stress, suicidal ideation, and protective factors in college students. Studies on Korean Youth, 23(3), 77-104.

34. McLaughlin, K. A., Hatzenbuehler, M. L. (2009). Stressful life events, anxiety sensitivity, and internalizing symptoms in adolescents. Journal of Abnormal Psychology, 118(3), 659. DOI 10.1037/a0016499.

35. Bingöl, T. Y., Batik, M. V., Hosoglu, R., Firinci Kodaz, A. (2019). Psychological resilience and positivity as predictors of self-efficacy. Asian Journal of Education and Training, 5(1), 63-69. DOI 10.20448/journal.522.

36. Liu, D., Zhou, Y., Li, G., He, Y. (2020). The factors associated with depression in schizophrenia patients: The role of self-efficacy, self-esteem, hope and resilience. Psychology, Health \& Medicine, 25(4), 457-469. DOI 10.1080/ 13548506.2019.1695862.

37. Liu, M. (2019). The relationship between emotional intelligence and coping style among the students in secondary vocational schools: Multiple mediation effects of resilience and general self-efficacy (Master's Thesis). Zhejiang University.

38. Cohen, S., Kamarck, T., Mermelstein, R. (1983). A global measure of perceived stress. Journal of Health and Social Behavior, 24(4), 385-396. DOI 10.2307/2136404.

39. Yang, T., Huang, H. (2003). An epidemiological study on the psychological pressure of urban residents in social transition. Chinese Journal of Epidemiology, 24(9), 760-764.

40. Connor, K. M., Davidson, J. R. (2003). Development of a new resilience scale: The Connor-Davidson resilience scale (CD-RISC). Depression and Anxiety, 18(2), 76-82. DOI 10.1002/(ISSN)1520-6394.

41. Yu, X. N., Zhang, J. X. (2007). A comparison between the Chinese version of ego-resiliency scale and ConnorDavidson resilience scale. Psychological Science, 44(5), 1169-1171. DOI 10.16719/j.cnki.1671-6981.2007.05.035.

42. Spitzer, R. L., Kroenke, K., Williams, J. B., Löwe, B. (2006). A brief measure for assessing generalized anxiety disorder: The GAD-7. Archives of Internal Medicine, 166(10), 1092-1097. DOI 10.1001/archinte.166.10.1092.

43. Caprara, G. V., Fida, R., Vecchione, M., Del Bove, G., Vecchio, G. M. et al. (2008). Longitudinal analysis of the role of perceived self-efficacy for self-regulated learning in academic continuance and achievement. Journal of Educational Psychology, 100(3), 525. DOI 10.1037/0022-0663.100.3.525.

44. Wen, S. F., Tang, D. L., Yu, G. L. (2009). The characteristics of regulatory emotional self-efficacy in Chinese graduate students. Psychological Science, 46(3), 666-668. DOI 10.16719/j.cnki.1671-6981.2009.03.025.

45. Podsakoff, P. M., MacKenzie, S. B., Lee, J. Y., Podsakoff, N. P. (2003). Common method biases in behavioral research: A critical review of the literature and recommended remedies. Journal of Applied Psychology, 88(5), 879. DOI 10.1037/0021-9010.88.5.879. 
46. Ren, W. (2021). A study on college graduates' employment problem in the context of big data based on the event of COVID-19. 2021 4th International Conference on Big Data and Education, pp. 88-91. New York. DOI 10.1145/ 3451400.3451414.

47. Tian, F., Tian, S., Li, H., Zhen, Y., Shao, J. (2020). Psychological symptoms of public under COVID-19 outbreak and improvement of emergency management system. China Safety Science Journal, 30(11), 30-36. DOI 10.16265/j.cnki.issn 1003-3033.2020.11.005.

48. Demerouti, E., Bakker, A. B., Nachreiner, F., Schaufeli, W. B. (2001). The job demands-resources model of burnout. Journal of Applied Psychology, 86(3), 499. DOI 10.1037/0021-9010.86.3.499.

49. Ran, L., Wang, W., Ai, M., Kong, Y., Chen, J. et al. (2020). Psychological resilience, depression, anxiety, and somatization symptoms in response to COVID-19: A study of the general population in China at the peak of its epidemic. Social Science \& Medicine, 262, 113261. DOI 10.1016/j.socscimed.2020.113261.

50. Hobfoll, S. E., Schumm, J. A. (2009). Conservation of resources theory: Application to public health promotion. In: R. J. DiClemente, R. A. Crosby, \& M. C. Kegler (Eds.), Emerging theories in health promotion practice and research, pp. 131-156. Jossey-Bass/Wiley.

51. Wen, W., Tang, D., Yu, G. (2009). Emotion regulation self-efficacy: A new research topic. Advances in Psychological Science, 58(4), 598-604. DOI 10.16719/j.cnki.1671-6981.2009.03.025. 